\title{
Synthesis and biological activity of 1,2,4-triazolo- [3,4-b]thiadiazole as antimicrobial agents
}

\author{
Piyush B. Vekariya, Jalpa R. Pandya, Vaishali Goswami, Hitendra S. Joshi* \\ Chemical Research Laboratory, Department of Chemistry, Saurashtra University, \\ Rajkot - 360005, India \\ *E-mail address: drhsjoshi49@gmail.com
}

\begin{abstract}
Some novel 6-fluoro chroman derivatives having 1,2,4-triazolo-[3,4-b]thiadiazole were synthesized and characterized by IR, NMR and mass spectral analysis. All synthesized compounds were screened for antimicrobial activity using broth dilution method. All the compounds showed good antimicrobial activity and compound 5e showed significant antibacterial activity.
\end{abstract}

Keywords: 6-Fluoro chroman; 1,2,4-triazole; triazolothiadiazole; antimicrobial activity

\section{INTRODUCTION}

Over the years, synthetic heterocyclic chemistry is providing momentum to the development of new drug scaffolds through interactive manipulation of functional groups around the basic skeleton. Among these, heterocyclic compounds have been given special importance because of a wide variety of biological properties associated with them. The importance of heterocycles in biological systems encouraged chemists to design and modify new heterocyclic compounds [1,2]. During the last two decades, the chemistry of 1,2,4triazole and 1,2,4-triazolo-[3,4-b]-1,3,4-thiadiazole and their derivatives have received considerable attention owing to their synthetic and effective biological importance [3-5]. 1,2,4-Triazoles and their derivatives occupy a essential position in medicinal chemistry because of their potential biological activities such as antibacterial [6], antifungal [7], antitubercular [8], anti-inflammatory [9] etc. The 1,2,4-triazole ring is an integral part found in various drugs such as rizatriptan, ribavirin, and fluconazole (Fig.1), which find a wide range of applications in pharmaceutical industry [10-12].

Our previous lab members have synthesized 2-(3'5'-dichlorobenzo[b]thiophen-2'yl)-5-arylamino-1,3,4-thiadiazoles [13] from triazole and some new thiosemicarbazide and 1,3,4-thiadiazole heterocycles bearing the benzo[b]thiophene nucleus [14] as potent antituberculer and antimicrobial agents. In light of wide varieties of therapeutic activities exhibited by thiadiazole, we have embarked upon the synthesis of some new thiadiazole derivatives which have been described in following sections. 


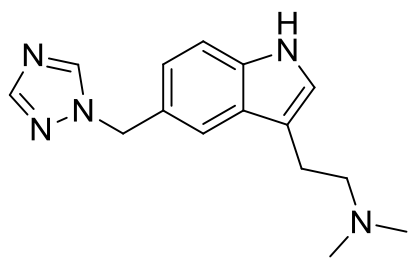

Rizatriptan<smiles>NC(=O)c1ncn([C@@H]2O[C@H](CO)[C@@H](O)[C@H]2O)n1</smiles>

Ribavirin

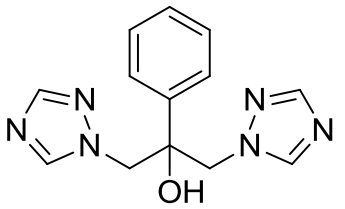

Fluconazol

Fig.1

\section{EXPERIMENTAL}

All chemicals and solvents were purchased from Spectrochem Pvt Ltd., Mumbai of LR grade and were used without further purification. Melting points were taken in open capillary method and are uncorrected. IR spectra were recorded on FTIR-8400 spectrophotometer (Shimadzu, Kyoto, Japan), using DRS probe KBr pallet. ${ }^{1} \mathrm{HNMR}$ spectra of the synthesized compounds were recorded on a Bruker-Avance-II (400 MHz) $\mathrm{CDCl}_{3}$ and DMSO-d $\mathrm{d}_{6}$ solvent. Chemical shifts are expressed in $\delta$ ppm downfield from TMS as an internal standard. Mass spectra were determined using direct inlet probe on a GCMS-QP 2010 mass spectrometer (Shimadzu, Kyoto, Japan).

To a stirred solution of methyl 6-fluorochroman-2-carboxylate 1, $(2.0 \mathrm{~g}, 0.01 \mathrm{~mol})$ in absolute ethanol $(25 \mathrm{ml})$ cooled at $(-5)^{\circ} \mathrm{C}$, hydrazine hydrate $(99 \%),(4.0 \mathrm{ml}, 0.08 \mathrm{~mol})$ was added and reaction mixture was allowed to stir at $0-(-5){ }^{\circ} \mathrm{C}$ for 10 hours. After the completion of reaction solid residue obtained was filtered, washed with cold ethanol and dried to afford 6-fluorochroman-2-carbohydrazide 2, Yield: $2.0 \mathrm{~g}$ (98\%).

To a stirred solution 6-fluorochroman-2-carbohydrazide $2(2.0 \mathrm{~g}, 0.1 \mathrm{~mol})$ and potassium hydroxide $(1.0 \mathrm{~g}, 0.15 \mathrm{~mol})$ in methanol $(25 \mathrm{ml})$, carbon disulphide $(11.4 \mathrm{~g}, 0.15$ mol) was added. Reaction mixture was allowed to stir for 22-24 hours at RT. After completion of reaction precipitate obtained was filtered, washed with diethyl ether and dried to afford potassium 2-[(6-fluorochroman-2-yl) carbonyl] hydrazine carbodithioate 3, Yield: $2.88 \mathrm{~g}(93 \%)$. There is no need to purify the salt for further reaction.

A mixture of potassium 2-[(6-fluorochroman-2-yl) carbonyl] hydrazine carbodithioate $3(3.5 \mathrm{~g}, 0.1 \mathrm{M})$ in water $(5 \mathrm{ml})$ and hydrazine hydrate $(3.4 \mathrm{ml}, 0.05 \mathrm{M})$ was refluxed for 6-7 $\mathrm{h}$ with occasional shaking. The colour of the reaction mixture changed to green with the evolution of hydrogen sulfide. A homogenous reaction mixture was obtained during the reaction process. The completion of the reaction was monitored on TLC. The reaction mixture was cooled to room temperature and diluted with water $(100 \mathrm{ml})$. On acidification with concentrated hydrochloric acid, the required triazole 4 gets precipitated. Further it was filtered, washed thoroughly with cold water and recrystallized from ethanol.

\section{1. General procedure for the preparation of 3-(6-Fluorochroman-2-yl)-6-aryl $[1,2,4]$ triazolo $[3,4-b][1,3,4]$ thiadiazoles.}

A mixture of 4-amino-5-(6-fluorochroman-2-yl)-4H-1,2,4-triazole-3-thiol (2.66 g, 0.01 mol) and different aryl acids $(0.01 \mathrm{~mol})$ in phosphorous oxychloride $(15 \mathrm{ml})$ was refluxed with continuous stirring. After completion the reaction (15-16 hours monitoring by TLC), the content was cooled to room temperature and was poured on crushed ice and thus solid 
separated out was filtered, washed with water and neutralized with sodium bicarbonate solution. Crude product was purified by column chromatography to give the analytical pure compounds. Physical constants of newly synthesized triazolo[3,4-b][1,3,4]thiadiazoles derivatives $5 \mathrm{a}-5 \mathrm{j}$ are recorded in Table 1.

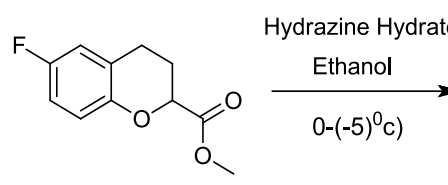

1<smiles>NNC(=O)C1CCc2cc(F)ccc2O1</smiles>

2

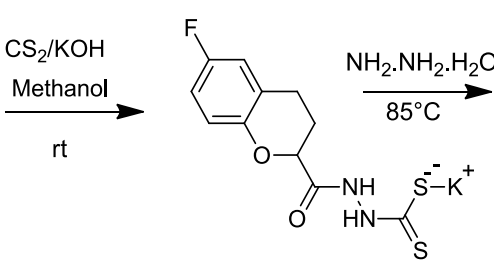

3



Where $\mathrm{R}=\mathrm{Aryl}$

Table 1. Physical Constant table of 1,2,4-triazolo-[3,4-b]thiadiazole derivatives (5a-5j).

\begin{tabular}{|c|c|c|c|c|c|}
\hline Sr. No & Compound & Substitution R & M. F. & M. W. & $\begin{array}{c}\text { Yield } \\
\text { (\%) }\end{array}$ \\
\hline 1 & $5 \mathrm{a}$ & $3-\mathrm{Cl} \mathrm{C} \mathrm{H}_{4}$ & $\mathrm{C}_{18} \mathrm{H}_{12} \mathrm{ClFN}_{4} \mathrm{OS}$ & 386.83 & 95 \\
\hline 2 & $5 \mathrm{~b}$ & $3,4-\mathrm{diOMe}_{6} \mathrm{H}_{3}$ & $\mathrm{C}_{20} \mathrm{H}_{17} \mathrm{FN}_{4} \mathrm{O}_{3} \mathrm{~S}$ & 412.43 & 79 \\
\hline 3 & $5 \mathrm{c}$ & $4-\mathrm{NH}_{2} \mathrm{C}_{6} \mathrm{H}_{4}$ & $\mathrm{C}_{18} \mathrm{H}_{14} \mathrm{FN}_{5} \mathrm{OS}$ & 367.40 & 87 \\
\hline 4 & $5 \mathrm{~d}$ & $4-\mathrm{NO}_{2} \mathrm{C}_{6} \mathrm{H}_{4}$ & $\mathrm{C}_{18} \mathrm{H}_{12} \mathrm{FN}_{5} \mathrm{O}_{3} \mathrm{~S}$ & 397.38 & 94 \\
\hline 5 & $5 \mathrm{e}$ & $2-\mathrm{NH}_{2} \mathrm{C}_{6} \mathrm{H}_{4}$ & $\mathrm{C}_{18} \mathrm{H}_{14} \mathrm{FN}_{5} \mathrm{OS}$ & 367.40 & 82 \\
\hline 6 & $5 \mathrm{f}$ & $2-\mathrm{Cl} \mathrm{C}_{6} \mathrm{H}_{4}$ & $\mathrm{C}_{18} \mathrm{H}_{12} \mathrm{ClFN}_{4} \mathrm{OS}$ & 386.83 & 81 \\
\hline 7 & $5 \mathrm{~g}$ & $4-\mathrm{Cl} \mathrm{Cl}_{6} \mathrm{H}_{4}$ & $\mathrm{C}_{18} \mathrm{H}_{12} \mathrm{ClFN}_{4} \mathrm{OS}$ & 386.83 & 74 \\
\hline
\end{tabular}




\begin{tabular}{|c|c|c|c|c|c|}
\hline 8 & $5 \mathrm{~h}$ & $2-\mathrm{CH}_{3} \mathrm{C}_{6} \mathrm{H}_{4}$ & $\mathrm{C}_{19} \mathrm{H}_{15} \mathrm{FN}_{4} \mathrm{OS}$ & 366.41 & 72 \\
\hline 9 & $5 \mathrm{i}$ & $3-\mathrm{NH}_{2} \mathrm{C}_{6} \mathrm{H}_{4}$ & $\mathrm{C}_{18} \mathrm{H}_{14} \mathrm{FN}_{5} \mathrm{OS}$ & 367.40 & 86 \\
\hline 10 & $5 \mathrm{j}$ & $4-\mathrm{OMeC}_{6} \mathrm{H}_{4}$ & $\mathrm{C}_{19} \mathrm{H}_{15} \mathrm{FN}_{4} \mathrm{O}_{2} \mathrm{~S}$ & 382.41 & 83 \\
\hline
\end{tabular}

\section{2. Analytical data}

\section{6-(3-Chlorophenyl)-3-(6-fluorochroman-2-yl)-[1,2,4] triazolo[3,4-b][1,3,4] thiadiazole}

(5a). mp 150-154 ${ }^{\circ} \mathrm{C}$; IR (DRS): 3073, 3031, 2957, 2847, 1625, 1462, 1442, 1325, 1258, 1140, 1065, 1018, 825, 748, 701, $685 \mathrm{~cm}^{-1} ;{ }^{1} \mathrm{H}$ NMR (400 MHz, $\left.\mathrm{CDCl}_{3}\right)$ : $\delta$ ppm 2.44-2.48 $(\mathrm{m}, 1 \mathrm{H}, 2 \mathrm{CH}), 2.74-2.76(\mathrm{~m}, 1 \mathrm{H}, 2 \mathrm{CH}), 3.04(\mathrm{~m}, 2 \mathrm{H}, 2 \mathrm{CH}), 5.66-5.68(\mathrm{~m}, 1 \mathrm{H}, \mathrm{CH}), 6.82-$ 6.84(m, 3H, ArH), 7.48-7.50(d, $J=5.79 \mathrm{~Hz}, 1 \mathrm{H}, \operatorname{ArH}), 7.56(\mathrm{~m}, 1 \mathrm{H}, \mathrm{ArH}), 7.73-7.75(\mathrm{~d}, J=$ $6.69 \mathrm{~Hz}, 1 \mathrm{H}, \mathrm{ArH}), 7.90(\mathrm{~s}, 1 \mathrm{H}, \mathrm{ArH}) . \mathrm{MS}: m / z=386[\mathrm{M}]^{+}$; Anal. Calcd for $\mathrm{C}_{18} \mathrm{H}_{12} \mathrm{ClFN}_{4} \mathrm{OS}$ : C, 55.89; H, 3.13; N, 14.48. Found: C, 55.83; H, 3.04; N, $14.08 \%$.

\section{6-(3,4-Dimethoxyphenyl)-3-(6-fluorochroman-2-yl)-[1,2,4]triazolo[3,4-b][1,3,4]}

thiadiazole (5b). $\mathrm{mp} 119-121{ }^{\circ} \mathrm{C}$; IR (DRS): 3090, 3020, 2935, 2839, 1637, 1492, 1440, $1363,1138,1058,1020,810,756,705,680 \mathrm{~cm}^{-1}$; ${ }^{1} \mathrm{H}$ NMR (400 MHz, DMSO): $\delta$ ppm 2.41$2.44(\mathrm{~m}, 1 \mathrm{H}, 2 \mathrm{CH}), 2.58-2.65(\mathrm{~m}, 1 \mathrm{H}, 2 \mathrm{CH}), 3.02-3.19(\mathrm{~m}, 2 \mathrm{H}, 2 \mathrm{CH}), 3.90\left(\mathrm{~s}, 6 \mathrm{H}, \mathrm{OCH}_{3}\right.$, $\left.\mathrm{OCH}_{3}\right), 5.70-5.73$ (d, d, $\left.J=4.4 \mathrm{~Hz}, 3.4 \mathrm{~Hz}, 1 \mathrm{H}, \mathrm{CH}\right), 6.78-6.93$ (m, 3H, ArH), 7.09-7.11 (d, $J$ $=8.44 \mathrm{~Hz}, 1 \mathrm{H}, \operatorname{ArH}), 7.39(\mathrm{~s}, 1 \mathrm{H}, \operatorname{ArH}), 7.49-7.51(\mathrm{~d}, J=7.72 \mathrm{~Hz}, 1 \mathrm{H}, \operatorname{ArH}) . \mathrm{MS}: m / z=412$ $[\mathrm{M}]^{+}$; Anal. Calcd for $\mathrm{C}_{20} \mathrm{H}_{17} \mathrm{FN}_{4} \mathrm{O}_{3} \mathrm{~S}$ : C, 58.24; H, 4.15; N, 13.58. Found: C, 58.18; H, 3.99; $\mathrm{N}, 13.49 \%$.

4-(3-(6-Fluorochroman-2-yl)-[1,2,4]triazolo[3,4-b][1,3,4]thiadiazol-6-yl)aniline (5c). mp 168-170 ${ }^{\circ} \mathrm{C}$; IR (DRS): 3422, 3378, 3030, 2964, 2853, 1642, 1612, 1581, 1471, 1368, 1247 , 1156, 1057, 1014, 819, 744, 710, $678 \mathrm{~cm}^{-1} ;{ }^{1} \mathrm{H}$ NMR (400 MHz, DMSO): $\delta$ ppm7.74-7.17 $(\mathrm{m}, 2 \mathrm{H}), 6.76-6.62(\mathrm{~m}, 4 \mathrm{H}), 6.50-6.48(\mathrm{dd}, 1 \mathrm{H}), 5.24-.5 .20(\mathrm{~s}, 2 \mathrm{H}), 5.16-5.13(\mathrm{t}, 1 \mathrm{H}), 3.17-$ $3.15(\mathrm{~m}, 2 \mathrm{H}), 2.56-2.54(\mathrm{~m}, 1 \mathrm{H}), 2.36-2.34(\mathrm{dq}, 1 \mathrm{H}) . \mathrm{MS}: \mathrm{m} / \mathrm{z}=367[\mathrm{M}]^{+}$; Anal. Calcd for $\mathrm{C}_{18} \mathrm{H}_{14} \mathrm{FN}_{5} \mathrm{OS}$ : C, 58.84; H, 3.84; N, 19.06. Found: C, 58.69; H, 3.78; N, $18.90 \%$.

3-(6-Fluorochroman-2-yl)-6-(4-nitrophenyl)-[1,2,4] triazolo[3,4-b][1,3,4]thiadiazole (5d). $\mathrm{mp} 158-160{ }^{\circ} \mathrm{C}$; IR (DRS): 3074, 2987, 2851, 1645, 1612, 1585, 1468, 1345, 1184, 1250 , 1061, 1023, 820, 780, 744, 695, $566 \mathrm{~cm}^{-1} ;{ }^{1} \mathrm{H}$ NMR (400 MHz, DMSO): $\delta$ ppm 8.42-8.40 (m, $2 \mathrm{H}), 8.07-8.05(\mathrm{~m}, 2 \mathrm{H}), 6.76-6.75(\mathrm{~m}, 2 \mathrm{H}), 6.50-6.49(\mathrm{dd}, 1 \mathrm{H}), 5.15-5.13(\mathrm{t}, 1 \mathrm{H}), 3.17-3.15$ $(\mathrm{m}, 2 \mathrm{H}), 2.52-2.50(\mathrm{dq}, 1 \mathrm{H}), 2.36-2.34(\mathrm{dq}, 1 \mathrm{H}) . \mathrm{MS}: \mathrm{m} / z=397[\mathrm{M}]^{+}$; Anal. Calcd for $\mathrm{C}_{18} \mathrm{H}_{12} \mathrm{FN}_{5} \mathrm{O}_{3} \mathrm{~S}: \mathrm{C}, 54.40 ; \mathrm{H}, 3.04 ; \mathrm{N}, 17.62$. Found: C, 54.28; H, 2.93; N, $17.44 \%$.

2-(3-(6-Fluorochroman-2-yl)-[1,2,4]triazolo[3,4-b][1,3,4]thiadiazol-6-yl)aniline (5e). mp 147-149 ${ }^{\circ} \mathrm{C}$; IR (DRS): 3442, 3091, 3081, 2975, 2844, 1641, 1579, 1556, 1464, 1357, 1242, 1145, 1088, 1017, 832, 750, $687 \mathrm{~cm}^{-1}$; ${ }^{1} \mathrm{H}$ NMR (400 MHz, DMSO): $\delta$ ppm 7.75-7.74 (dd, $1 \mathrm{H}), 7.22-7.20(\mathrm{td}, 1 \mathrm{H}), 7.01-7.00(\mathrm{~m}, 2 \mathrm{H}), 6.75-6.73(\mathrm{~m}, 2 \mathrm{H}), 6.50-6.49(\mathrm{dd}, 1 \mathrm{H}), 5.59-5.57(\mathrm{~s}$, $2 \mathrm{H}), 5.26-5.24(\mathrm{t}, 1 \mathrm{H}), 3.17-3.15(\mathrm{~m}, 2 \mathrm{H}), 2.61-2.60(\mathrm{dq}, 1 \mathrm{H}), 2.34-2.32(\mathrm{dq}, 1 \mathrm{H}) . \quad \mathrm{MS}: \mathrm{m} / z=$ 
$367[\mathrm{M}]^{+}$; Anal. Calcd for $\mathrm{C}_{18} \mathrm{H}_{14} \mathrm{FN}_{5} \mathrm{OS}$ : C, 58.84; H, 3.84; N, 19.06. Found: $\mathrm{C}, 58.41 ; \mathrm{H}$, $3.78 ; \mathrm{N}, 18.99 \%$.

\section{6-(4-Chlorophenyl)-3-(6-fluorochroman-2-yl)-[1,2,4]triazolo[3,4-b][1,3,4]thiadiazole}

(5f). $\mathrm{mp} 116-118{ }^{\circ} \mathrm{C}$; IR (DRS): 3080, 2983, 2867, 1629, 1572, 1525, 1462, 1381, 1245, 1196, 1046, 1011, 830, 778, 701, 665, $578 \mathrm{~cm}^{-1}$; ${ }^{1} \mathrm{H}$ NMR (400 MHz, DMSO): $\delta$ ppm 7.68$7.65(\mathrm{~m}, 2 \mathrm{H}), 7.44-7.43(\mathrm{~m}, 2 \mathrm{H}), 6.74-6.72(\mathrm{~m}, 2 \mathrm{H}), 6.49-6.48(\mathrm{dd}, 1 \mathrm{H}), 5.26-5.24(\mathrm{t}, 1 \mathrm{H})$, 3.17-3.16 (m, 2H), 2.60-2.58 (dt,1H), 2.35-2.34 (dq,1H) MS: $m / z=386[\mathrm{M}]^{+}$; Anal. Calcd for $\mathrm{C}_{18} \mathrm{H}_{12} \mathrm{ClFN}_{4} \mathrm{OS}$ : C, 55.89; H, 3.13; N, 14.48. Found: C, 55.84; H, 2.97; N, $14.17 \%$.

\section{6-(2-Chlorophenyl)-3-(6-fluorochroman-2-yl)-[1,2,4]triazolo[3,4-b][1,3,4]thiadiazole}

(5g). mp 183-185 ${ }^{\circ} \mathrm{C}$; IR (DRS): 3077, 2978, 2863, 1625, 1609, 1563, 1464, 1331, 1238, $1142,1038,1014,870,832,778,668,514 \mathrm{~cm}^{-1}$; ${ }^{1} \mathrm{H}$ NMR (400 MHz, DMSO): $\delta$ ppm 8.00$7.98(\mathrm{~m}, 2 \mathrm{H}), 7.65-7.63(\mathrm{~m}, 2 \mathrm{H}), 6.76-6.74(\mathrm{~m}, 2 \mathrm{H}), 6.50-6.48(\mathrm{dd}, 1 \mathrm{H}), 5.16-5.14(\mathrm{t}, 1 \mathrm{H})$, 3.17-3.15 (m, 2H), 2.58-2.56 (m, 1H), 2.36-2.34 (dq,1H). MS: $m / z=386[\mathrm{M}]^{+}$; Anal. Calcd $\mathrm{C}_{18} \mathrm{H}_{12} \mathrm{ClFN}_{4} \mathrm{OS}$ : C, 55.89; H, 3.13; N, 14.48. Found: C, 55.67; H, 3.01; N, $14.21 \%$.

3-(6-Fluorochroman-2-yl)-6-(o-tolyl)-[1,2,4]triazolo[3,4-b][1,3,4]thiadiazole $\quad(6 \mathrm{~h}) . \mathrm{mp}$ 160-162 ${ }^{\circ} \mathrm{C}$; IR (DRS): 3063, 2962, 2854, 1603, 1545, 1542, 1452, 1325, 1260, 1146, 1060 , 1023, 812, 754, 662, $518 \mathrm{~cm}^{-1}$; ${ }^{1} \mathrm{H}$ NMR (400 MHz, DMSO): $\delta$ ppm 7.80-7.79 (m, $\left.1 \mathrm{H}\right), 7.40-$ $7.38(\mathrm{~m}, 4 \mathrm{H}), 6.75-6.73(\mathrm{~m}, 3 \mathrm{H}), 6.50-6.48(\mathrm{dd}, 1 \mathrm{H}), 5.26-5.24(\mathrm{t}, 1 \mathrm{H}), 3.17-.15(\mathrm{~s}, 3 \mathrm{H}), 2.61-$ $2.60(\mathrm{dq}, 1 \mathrm{H}), 2.34-2.33(\mathrm{dq}, 1 \mathrm{H}) . \mathrm{MS}: \mathrm{m} / z=366[\mathrm{M}]^{+}$; Anal. Calcd for $\mathrm{C}_{19} \mathrm{H}_{15} \mathrm{FN}_{4} \mathrm{OS}: \mathrm{C}$, 62.28; H, 4.13; N, 15.29. Found: C, 62.19; H, 3.97; N, $15.24 \%$.

3-(3-(6-Fluorochroman-2-yl)-[1,2,4]triazolo[3,4-b][1,3,4]thiadiazol-6-yl)aniline (5i). $\mathrm{mp}$ 109-111 ${ }^{\circ} \mathrm{C}$; IR (DRS): 3428, 3392, 3075, 2964, 2853, 1721, 1601, 1581, 1423, 1371, 1241, $1149,1054,1026,888,848,766,720,665,578 \mathrm{~cm}^{-1} ;{ }^{1} \mathrm{H}$ NMR (400 MHz, DMSO): $\delta$ ppm 7.71-7.59 (m, 2H), $7.27(\mathrm{t}, 1 \mathrm{H}), 6.83(\mathrm{dt}, 1 \mathrm{H}), 6.76-6.66(\mathrm{~m}, 2 \mathrm{H}), 5.19(\mathrm{t}, 1 \mathrm{H}), 4.26(\mathrm{~s}, 2 \mathrm{H})$, 3.20-3.03 (m, 2H), 2.51 (dq, 1H), 2.35 (dq, 1H.) MS: $\mathrm{m} / z=367[\mathrm{M}]^{+}$; Anal. Calcd for $\mathrm{C}_{18} \mathrm{H}_{14} \mathrm{FN}_{5} \mathrm{OS}$ : C, 58.84; H, 3.84; N, 19.06. Found: C, 58.53; H, 3.71; N, $18.90 \%$.

3-(6-Fluorochroman-2-yl)-6-(4-methoxyphenyl)-[1,2,4]triazolo[3,4-b][1,3,4] thiadiazole (5j). mp 224-226 ${ }^{\circ} \mathrm{C}$; IR (DRS): 3061, 2951, 2872, 1689, 1589, 1579, 1462, 1354, 1208, 1135, 1099, 1003, 819, 755, $688 \mathrm{~cm}^{-1} ;{ }^{1} \mathrm{H}$ NMR (400 MHz, DMSO): $\delta$ ppm $7.82(\mathrm{dt}, 1 \mathrm{H})$, $7.65(\mathrm{t}, 1 \mathrm{H}), 7.38(\mathrm{t}, 1 \mathrm{H}), 6.89(\mathrm{dt}, 1 \mathrm{H}), 6.76-6.65(\mathrm{~m}, 2 \mathrm{H}), 6.44(\mathrm{dd}, 1 \mathrm{H}), 5.15(\mathrm{t}, 1 \mathrm{H}), 3.79(\mathrm{~s}$, $3 \mathrm{H}), 3.17(\mathrm{~m}, 2 \mathrm{H}), 2.52(\mathrm{dq}, 1 \mathrm{H}), 2.36(\mathrm{dq}, 1 \mathrm{H})$. MS: $\mathrm{m} / z=382[\mathrm{M}]^{+}$; Anal. Calcd for $\mathrm{C}_{19} \mathrm{H}_{15} \mathrm{FN}_{4} \mathrm{O}_{2} \mathrm{~S}$ : C, 59.67; H, 3.95; N, 14.65. Found: C, 59.08; H, 3.88; N, $14.62 \%$.

\section{ANTI MICROBIAL ACTIVITY}

All the glass apparatus used were sterilized before use. The broth dilution technique was used to determine the minimum inhibitory concentration (MIC) of the synthesized compounds. Bacterial strain of Staphylococcus aureus, Streptococcus pyogenes, Pseudomonas aeruginosa and Escherichia coli and fungal strains of Aspergillus niger, Candida albicans and Aspergillus clavatus were used in the present study. DMSO was used as the solvent for the compounds. A blank test was carried out to check the antimicrobial activity of DMSO. 
Ampicillin and Chloramphenicol were used as the standard drugs for antibacterial activity. Greseofulvin was used as the standard drug for antifungal activity. The synthesized 1,2,4-triazolo-[3,4-b]thiadiazole 5a-5j were screened for their antimicrobial activity by the broth dilution method to evaluate the minimum inhibitory concentration Table 2.

Table 2. Antimicrobial activity of 1,2,4-triazolo-[3,4-b]thiadiazole derivatives (5a-5j).

\begin{tabular}{|c|c|c|c|c|c|c|c|}
\hline \multirow{4}{*}{$\begin{array}{l}\text { Sr. } \\
\text { No. }\end{array}$} & \multicolumn{4}{|c|}{ Antibacterial Activity } & \multicolumn{3}{|c|}{ Antifungal activity } \\
\hline & \multicolumn{4}{|c|}{ Minimal bactericidal concentration $\mu \mathrm{g} / \mathrm{ml}$} & \multirow{2}{*}{\multicolumn{3}{|c|}{$\begin{array}{l}\text { Minimal fungicidal concentration } \\
\qquad \mu \mathrm{g} / \mathrm{ml}\end{array}$}} \\
\hline & \multicolumn{2}{|c|}{ Gram +ve Bacteria } & \multicolumn{2}{|c|}{ Gram-ve Bacteria } & & & \\
\hline & $\begin{array}{c}\mathrm{S} . \\
\text { aureus }\end{array}$ & $\begin{array}{c}\text { S. } \\
\text { pyogenus }\end{array}$ & $\begin{array}{c}\text { E. } \\
\text { coli }\end{array}$ & $\begin{array}{c}\mathrm{P} . \\
\text { aeruginosa }\end{array}$ & C. albicans & $\begin{array}{c}\text { A. } \\
\text { niger }\end{array}$ & $\begin{array}{c}\text { A. } \\
\text { clavatus }\end{array}$ \\
\hline $5 \mathrm{a}$ & 100 & 62.5 & 250 & 250 & 1000 & $>1000$ & $>1000$ \\
\hline $5 b$ & 250 & 250 & 250 & 200 & $>1000$ & $>1000$ & $>1000$ \\
\hline $5 c$ & 125 & 100 & 250 & 100 & $>1000$ & $>1000$ & $>1000$ \\
\hline $5 \mathrm{~d}$ & 200 & 200 & 100 & 200 & 1000 & $>1000$ & $>1000$ \\
\hline $5 e$ & 62.5 & 125 & 62.5 & 100 & 500 & 1000 & 1000 \\
\hline $5 f$ & 125 & 200 & 100 & 125 & $>1000$ & 250 & 500 \\
\hline $5 g$ & 500 & 500 & 250 & 200 & 500 & 1000 & 1000 \\
\hline $5 \mathrm{~h}$ & 500 & 250 & 200 & 100 & $>1000$ & $>1000$ & $>1000$ \\
\hline $5 \mathrm{i}$ & 500 & 500 & 100 & 100 & 1000 & 250 & 250 \\
\hline $5 \mathrm{j}$ & 250 & 500 & 250 & 250 & 500 & 500 & 500 \\
\hline
\end{tabular}

MINIMAL INHIBITION CONCENTRATION

\begin{tabular}{|c|c|c|c|c|}
\hline \multirow{2}{*}{ Standard Drugs } & S. aureus & S. pyogenus & E. coli & P. aeruginosa \\
\cline { 2 - 5 } & \multicolumn{4}{|c|}{ (microgramme/ml) } \\
\hline Ampicillin & 250 & 100 & 100 & 100 \\
\hline Chloramphenicol & 50 & 50 & 50 & 50 \\
\hline
\end{tabular}

MINIMAL FUNGICIDAL CONCENTRATION

\begin{tabular}{|c|c|c|c|}
\hline \multirow{2}{*}{ Standard Drugs } & C. Albicans & A. Niger & A. Clavatus \\
\cline { 2 - 4 } & \multicolumn{3}{|c|}{ (microgramme/ml) } \\
\hline Greseofulvin & 500 & 100 & 100 \\
\hline
\end{tabular}

All of the precursors (5a-j) of the title compounds showed antibacterial activity in the range of $62.5-500 \mu \mathrm{g} / \mathrm{mL}$ for Staphylococcus aureus, $100-500 \mu \mathrm{g} / \mathrm{mL}$ for Streptococcus pyogenes, $100-500 \mu \mathrm{g} / \mathrm{mL}$ for Pseudomonas aeruginosa, and $62.5-500 \mu \mathrm{g} / \mathrm{mL}$ for Escherichia coli. It was observed that compound $5 \mathrm{e}(\mathrm{MIC}=62.5 \mu \mathrm{g} / \mathrm{mL})$ against $s$. aureus as well as compound $5 \mathrm{e}(\mathrm{MIC}=62.5 \mu \mathrm{g} / \mathrm{mL})$ against E.coli have found to be better active as 
compared to ampicillin ( $\mathrm{MIC}=250 \mu \mathrm{g} / \mathrm{mL}$ ). Except compound $5 \mathrm{e}, 5 \mathrm{a}$ and $5 \mathrm{c}$ compounds $5 \mathrm{~b}$, $5 \mathrm{f}, 5 \mathrm{~d}, 5 \mathrm{~g}$ and $5 \mathrm{i}$ were found moderately active against $S$. aureus, $p$. aeruginosa and Escherichia coli as compared to ampicillin. Against fungal pathogen $C$. albicans $5 \mathrm{~g}, 5 \mathrm{e}$ and $5 \mathrm{j}$ have shown good activity as compared to griseofulvin (MIC $=500 \mu \mathrm{g} / \mathrm{ml}$ ).

\section{CONCLUSION}

In present report, we are reporting very efficient method for the synthesis of some novel 1,2,4-triazolo-[3,4-b]thiadiazole derivatives possessing 6-fluoro chroman nucleus. All synthesized compounds were obtained in good yield. From the results of antimicrobial data, compounds 5e showed excellent results against Gram positive and Gram negative bacteria while compounds 5a, 5c and $5 \mathrm{f}$ were found moderate active. All synthesized compounds showed minimal activity against fungi pathogens as compared to the standard drugs.

\section{ACKNOWLEDGMENT}

Authors are thankful to Department of Chemistry for providing laboratory facilities. The authors are also thankful for the facilities and grants given under UGC-SAP for Department Research Support (DRS) and Department of Science and Technology (DST) New Delhi for Fund for Improvement of Science and Technology (FIST). Authors are also thankful to RSIC Chandigarh for providing ${ }^{1} \mathrm{H}$ NMR \& ${ }^{13} \mathrm{C}$ NMR spectral analysis of the compounds.

\section{References}

[1] R. K. Tiwari, et al., Bioog Med Chem Lett. 16 (2006) 413.

[2] P. Kumar, A. Kumar, J. K. Makrandi, J. Heterocyclic Chem. (2013).

[3] J. G. Sadaf, A. K. Suroor, S. Nadeem, Bioorg. Med. Chem. Lett. 20 (2010) 4762-4765.

[4] L. A. Gabriela, F. B. Stefania, B. Gabriela, S. Ioana, S. Gabriel, D. Constantin, Eur. J. Med. Chem. 45 (2010) 6139-6146.

[5] V. Padmavathi, G. Sudhakar Reddy, A. Padmaja, P. Kondaiah, P. Ali-Shazia, Eur. J. Med. Chem. 44 (2009) 2106-2112.

[6] I. R. Ezabadi, C. Camoutsis, P. Zoumpoulakis, A. Geronikaki, M. Sokovic, J. Glamocilija, A. Ciric, Bioorg Med Chem. 16 (2008) 1150.

[7] L. Meerpoel, et al., J Med Chem. 48 (2005) 2184.

[8] K. Walczak, A. Gondela, J. Suwinski, Eur. J. Med. Chem. 39 (2004), 849.

[9] L. Labanauskas, V. Kalcas, E. Udrenaite, P. Gaidelis, A. BrukĐtus, Farmaco 59 (2004) 255.

[10] D. Bianchi, P. Cesti, S. Spezia, C. Garavaglia, L. Mirenna, Chemoenzymic J. Agric. Food Chem. 39 (1991) 197.

[11] T. Chen, C. Dwyre-Gygax, S. T. Hadfield, C. Willetts, C. Breuil, J. Agric. Food Chem. 44 (1996) 1352. 
[12] J. L. Garca Ruano, M. Cifuentes Garca, A. M. Martn Castro, J. H. Rodrguez Ramos, Org. Lett. 4 (2002) 55.

[13] K. M. Thaker, H. S. Joshi, I. J. Chem. 36(23) (2005).

[14] S. L.Vasoya, D. J. Paghdar, P. T. Chovatia, H. S. Joshi, Journal of sciences, Islamic Republic of Iron 16(1) (2005) 33-36.

( Received 16 January 2014; accepted 21 January 2014 ) 